RECyT

Año 22 / № 33 / 2020 / 76-84

\title{
Glicósidos de esteviol y compuestos fenólicos en infusiones de Stevia rebaudiana dependiendo de la variedad
}

\author{
Steviol glycosides and phenolic compounds in Stevia rebaudiana infusions and \\ depending on the variety
}

Liliana Celaya ${ }^{1, *}{ }^{*}$ Darío Taiariol², Silvia Valle ${ }^{3}$, Nicolás Kolb Koslobsky ${ }^{1}$

1- CONICET-UNaM, Facultad de Ciencias Exactas Químicas y Naturales, Universidad Nacional de Misiones, Posadas, Argentina.

2- Estación Experimental Agropecuaria INTA Bella Vista, Bella Vista, Corrientes, Argentina.

3- Finca Experimental La Posta perteneciente a LATSER S.A. - Cámara del Tabaco de Jujuy.

*E- mail: lilianacelaya@fceqyn.unam.edu.ar

Recibido el 10 de septiembre de 2019, Aprobado el 14 de enero de 2020.

\section{Resumen}

El objetivo de este estudio fue caracterizar infusiones de tres variedades de Stevia rebaudiana (stevia) cultivadas en Argentina determinando la recuperación de sólidos totales, de glicósidos de esteviol y de compuestos fenólicos antioxidantes. La recuperación de sólidos totales en las infusiones estuvo entre $45-63 \%$ de las hojas mientras que la recuperación de glicósidos de esteviol estuvo entre 13-17\%. Por otro lado los compuestos fenólicos recuperados estuvieron entre $2-8 \%$ de las hojas. Las infusiones de hojas de las tres variedades de stevia presentan elevados contenidos de compuestos fenólicos y actividad antiradicalaria elevada frente a DPPH $\left(\right.$ IC $_{50}=18,6-27,7 \mu \mathrm{g} /$ $\mathrm{mL}$ ). En las infusiones de stevia se determinó la existencia de correlación elevada entre glicósidos de esteviol totales y sólidos totales, entre Rebaudiósido A y compuestos fenólicos totales, entre Esteviósido y taninos totales cuantificados. En cuanto al poder edulcorante, son necesarios de 0,2-0,6 g de hojas de stevia para endulzar una infusión (dependiendo de la variedad). En general, infusiones de stevia con mayores contenidos de glicósidos de esteviol totales proporcionan mayores contenidos de compuestos fenólicos antioxidantes.

Palabras clave: Stevia rebaudiana; Infusión; Glicósidos de esteviol; Compuestos fenólicos.

\section{Abstract}

The aim of this study was to characterize three Stevia rebaudiana (stevia) infusions that grow in Argentina in order to determine the overall solids recovery, steviol glycosides and phenolic compounds with antiradical activity. That total infusion retrievals on dry leaves varied from 45 to $53 \%$. The steviol glycosides recovery was between 13 and $17 \%$ while the phenolic compounds were around 2 to $8 \%$. The three varieties of stevia infusions show high contents of phenolic compounds and strong scavenging activity against DPPH• (IC50= 18.6-27.7 $\mu \mathrm{g} / \mathrm{mL})$.

High correlations were found in the stevia infusions between steviol glycosides and total solids, Rebaudióside A and total phenolic compounds and between Stevioside and tannin contents. Respect to the stevia sweetness potency and variety, 0.2-0.6 g of leaves, are necessary to sweeten a cup of the infusion. In general, stevia infusions with higher steviol glycosides provide more antiradical phenolic compounds.

Keywords: Stevia rebaudiana; Infusion; Steviol glycosides; Phenolic compounds.

\section{Introducción}

Stevia rebaudiana Bertoni (stevia), es una hierba originaria de Paraguay, actualmente cultivada en diversos países alrededor del mundo. El mayor valor de esta planta está en los fitoquímicos dulces que se extraen de sus hojas, llamados glicósidos de steviol (o steviol glicósidos), SGs [1]. Los SGs de stevia han sido aprobados por el Comité Mixto de Expertos en Aditivos Alimentarios de la FAO/OMS [2], en el año 2008 para ser utilizados como aditivos y se emplean actualmente como edulcorantes en todo el mundo. Por su parte las hojas de stevia están aprobadas para ser utilizadas como alimento en diversos países incluyendo Argentina [3].

Hasta la actualidad, se han identificado más de 30 SGs en $S$. rebaudiana [2-6]. Las hojas de la planta contienen en total, de 4 a 20\% de SGs totales (expresados en base seca). Esteviósido (Stv), Rebaudiósido A (RbA) y Rebaudiósido $\mathrm{C}(\mathrm{RbC})$ son los SGs de mayor presencia en stevia.

En las últimas décadas se han desarrollado variedades mejoradas de stevia con enfoque en la cantidad y calidad de los SGs presentes en las hojas [5, 7-9]. En general, las variedades mejoradas contienen concentraciones de SGs 
totales elevadas y relaciones $\mathrm{RbA} / \mathrm{Stv}$ altas. Con relaciones $\mathrm{RbA} / \mathrm{Stv}$ altas se reducen retrogustos indeseables en extractos de stevia $[1,10]$. Además de RbA, otros SGs deseables en hojas y extractos son RbC y Rebaudiósido D. RbC ha sido reportado como resaltador del sabor dulce en mezclas de SGs utilizadas como edulcorantes [11]. Rebaudiósido D por su parte, con sabor dulce más puro en comparación con otros SGs y en comparación con sacarosa, se encuentra presente en hojas de stevia en porcentajes muy bajos hasta la actualidad [12].

Además de los SGs, otros fitoquímicos presentes en hojas de stevia, son monosacáridos y fibra cruda, compuestos fenólicos, fitoesteroles y triterpenos, proteínas, minerales, clorofila, resinas, ácidos orgánicos, ácidos grasos y aceite esencial [5,13-14]. Particularmente, en el caso de las hojas de stevia es frecuente realizar la cuantificación de las sustancias solubles en agua, de los carbohidratos totales ( ${ }^{\circ}$ Brix) o bien de los sólidos totales (o extracto seco) para completar la caracterización de las hojas o de sus extractos acuosos [17-21]. Estas determinaciones proporcionan una primera aproximación del contenido de SGs en stevia [21-23].

Además de los edulcorantes presentes en las hojas de stevia, estas contienen compuestos fenólicos con elevada actividad antioxidante [6, 24-25]. Sobre este hecho se basa el interés creciente en el uso de hojas o extractos en comparación con los SGs purificados de stevia. Los flavonoides identificados previamente en stevia incluyen quercetina, kaeferol, apigenina, luteolina y sus derivados glicosilados [6, 26-27]; entre los ácidos fenólicos se identificaron ácidos clorogénicos, ácido p-cumárico, ácido rosmarínico, ácido ferúlico, ácido caféico y diversos derivados glicosilados de los mismos.

En relación al contenido de compuestos fenólicos de hojas o extractos de stevia existen reportado en la literatura científica muy diversos trabajos [7], estos incluyen el perfil de compuestos fenólicos, los contenidos de compuestos fenólicos totales y la actividad antioxidante. Otros estudios han establecido correlaciones entre los perfiles de compuestos fenólicos y determinados SGs en extractos de stevia [25, 28, 29]. Estos estudios han sido realizados en variedades o cultivares específicos de stevia, al igual que las investigaciones anteriores realizadas sobre infusiones de hojas de la planta $[18,30,31]$. Poco se conoce sobre las relaciones que existen entre los contenidos de compuestos fenólicos antioxidantes, los contenidos de SGs y los contenidos de sólidos totales en infusiones de stevia de variedades cultivadas en Argentina.

El objetivo del presente estudio fue determinar la recuperación de sólidos totales (STE), de SGs totales (SGT), de compuestos fenólicos antioxidantes (FT) y de taninos totales (TT) en infusiones de stevia de variedades cultivadas en Argentina. Se establecieron además las relaciones existentes entre STE, FT, TT y SGs, dependiendo de la variedad.

\section{Materiales y Métodos}

\section{Reactivos y Solventes}

Los reactivos y solventes utilizados se obtuvieron de varios proveedores: el etanol fue Cicarelli (Reagents S.A., San Lorenzo, Argentina). El ácido acético, agua, acetonitrilo y etanol grado HPLC fueron de Merck (Darmstadt, Alemania). El AG (ácido gálico) y DPPH• (2,2-difenil-1-picrilhidrazilo) de Sigma-Aldrich (Steinheim, Alemania); $\mathrm{NaHCO}_{3}$, BHT (butilhidroxitolueno), PVPP (polivinilpolipirrolidona) y Folin-Ciocalteu fueron Anedra (Tigre, Argentina). Los estándares de Stv (98,5\%), RbA (99,5\%) y RbC (99,5\%) fueron obtenidos dentro del Proyecto 16Q624 y chequeados con solución estándar de glicósidos de steviol calidad USP de Sigma-Aldrich (Steinheim, Alemania). El agua utilizada fue agua bidestilada y deionizada.

\section{Material Vegetal}

Se utilizaron hojas de stevia de tres cultivares experimentales. La elección de estos cultivares se basó en que corresponden a las variedades de stevia que se entregan a los productores agrícolas en cada región; además, las condiciones de cultivo utilizadas en cada caso son las que más se asemejan a las utilizadas por los productores en cada región. Stevia SK (variedad mejorada 16Q511-K-C1) fue de un cultivo del Proyecto UNaM-16Q511 (Posadas, Misiones). Stevia SE fue del cultivar E-L8, Estación Experimental Bella Vista (Bella Vista, Corrientes). Stevia SC fue de un cultivo experimental de "La Posta", Cooperativa de Tabacaleros de Jujuy (Perico, Jujuy). Las muestras representativas para este estudio fueron cosechadas en enero de 2017 (cosecha de verano) de poblaciones homogéneas de 1,0 ha (cultivar SK), 0,5 ha (cultivar SE) y 0,5 ha (cultivar SC). La fenología de la planta en el momento de la cosecha fue a inicios de la floración, coincidente con los contenidos máximos de SGs y con los porcentajes más altos de RbA en las hojas [8, 21, 23, 32].

Las muestras cosechadas se secaron al sol por 4-5 días, separando las hojas que se molieron hasta un tamaño de 20 mesh y se guardaron en freezer hasta su procesamiento analítico.

El contenido de humedad se determinó conjuntamente con los ensayos, secando muestras representativas (2-3 g, $102 \pm 2^{\circ} \mathrm{C}$ ), hasta peso constante. El control utilizado fue té negro "Amanda" (Posadas, Argentina), sometido al mismo procesamiento analítico que las hojas de stevia.

\section{Preparación de infusiones y caracterización}

Las infusiones se prepararon siguiendo la metodología descripta por Rao y colaboradores [31], con mínimas modificaciones. Diferentes pesos de hojas de stevia (0,2 a 2,0 g) 
se pusieron en contacto con $200 \mathrm{~mL}$ de agua a temperatura de ebullición por $5 \mathrm{~min}$. Para preparar las infusiones de té negro se utilizaron 2,0 g de té negro en $200 \mathrm{~mL}$ de agua a ebullición ( $5 \mathrm{~min}$ ). Las infusiones se realizaron por triplicado. Las infusiones resultantes se filtraron y se mantuvieron en la oscuridad $\left(4-5^{\circ} \mathrm{C}\right)$ hasta su procesamiento analítico.

Los contenidos de sólidos totales (STE) en las infusiones de determinaron secando $10 \mathrm{~mL}$ de cada infusión (en platillo de acero previamente pesado) a $102 \pm 2^{\circ} \mathrm{C}$, hasta peso constante. Los resultados obtenidos se expresaron como concentración (mg STE por infusión, $200 \mathrm{~mL}$ ) y recuperación (\% de sólidos recuperados a partir de $100 \mathrm{~g}$ de hojas secas).

\section{Cuantificación de SGs en hojas en las infusiones}

El contenido de SGs en hojas e infusiones de analizó por cromatografía líquida de alta performance, HPLC siguiendo la metodología propuesta por Kolb y colaboradores [8]. Para la cuantificación de SGs en las infusiones, se mezclaron partes iguales de infusión y etanol, se centrifugó por 5 min y el sobrenadante se utilizó para el análisis. Los análisis HPLC se realizaron en un cromatógrafo LC-20A (Shimadzu Corporation, Kyoto, Japón). Las condiciones de operación fueron: columna Agilent Zorbax $\mathrm{NH}_{2}(25,0$ $\times 0,46 \mathrm{~cm} ; 5 \mu \mathrm{m})$ trabajando a temperatura ambiente; detector UV a $210 \mathrm{~nm}$; fase móvil acetonitrilo: agua 80:20 (v/v) y pH 5 . El volumen de inyección fue de $5 \mu \mathrm{L}$. Para la calibración, se utilizaron soluciones de estándares entre los 0,2-1,0 g/L (Stv, RbA, RbC) en etanol: agua 70:30 (p/p).

Los resultados se expresaron como concentración (mg SG por infusión, 200mL), rendimiento (\% de SGs presentes extraídos), recuperación (\% de SGs recuperados a partir de $100 \mathrm{~g}$ de hojas secas).

\section{Determinación de compuesto fenólicos totales en las infusiones}

El contenido total de compuestos fenólicos (FT) se determinó con el reactivo Folin-Ciocaulteau [33], mezclando $25 \mu \mathrm{L}$ de infusión con $25 \mu \mathrm{L}$ de Folin-Ciocaulteau y $950 \mu \mathrm{L}$ de $\mathrm{NaHCO}_{3}(0,15 \%)$. Las mezclas de reacción se mantuvieron en la oscuridad por 60 min y luego se midió la absorbancia a $750 \mathrm{~nm}$. Los resultados de FT se determinaron utilizando una curva de calibración realizada con AG en diferentes concentraciones. Los valores de FT obtenidos se expresaron como concentración en mg de ácido gálico equivalente en la infusión (mg AGE /infusión, $200 \mathrm{~mL}$ ) y recuperación (\% AGE recuperados a partir de $100 \mathrm{~g}$ de hojas secas).

\section{Contenido de taninos totales en las infusiones}

Los contenidos de taninos totales (TT) se cuantificaron en las muestras siguiendo la metodología propuesta por
Azrul y colaboradores en 2014 [34], con mínimas modificaciones. Previamente se preparó una solución de PVPP en agua al $10 \% ; 500 \mu \mathrm{L}$ de esta solución se agregaron a 1000 $\mu \mathrm{L}$ de la mezcla de reacción FT. El set de tubos se agitó con vortex por 2 min y luego se mantuvo en la oscuridad $\left(4-5^{\circ} \mathrm{C}\right)$ por $15 \mathrm{~min}$. Los tubos se agitaron con vortex nuevamente y se centrifugaron (2000 rpm por $10 \mathrm{~min}$ ). La absorbancia a $750 \mathrm{~nm}$, se midió en el sobrenadante en cada caso. Los valores de TT se calcularon por diferencia restando los valores de absorbancia de FT [34]. Las mismas determinaciones se repitieron en el té negro utilizado como control.

\section{Actividad antioxidante de las infusiones}

Para determinar la actividad antioxidante in vitro se utilizaron las infusiones obtenidas con $2 \mathrm{~g}$ de hojas en 200 $\mathrm{mL}$ de agua. Estas infusiones se diluyeron a $2 \mathrm{mg} / \mathrm{mL}$ para determinar la actividad antiradicalaria frente a DPPH • [35]; $50 \mu \mathrm{L}$ de diluciones convenientes de infusiones (50 a 2000 $\mu \mathrm{g} / \mathrm{mL}$ ), se mezclaron con $1000 \mu \mathrm{L}$ de solución de DPPH• $(0,31 \mathrm{mM})$ en metanol dejando la mezcla reaccionar por 30 min en la oscuridad, midiendo la absorbancia de la mezcla reaccionante a $515 \mathrm{~nm}$. La concentración de infusión que reduce $\mathrm{DPPH} \bullet$ a la mitad, $\mathrm{IC}_{50}(\mu \mathrm{g} / \mathrm{mL})$ se calculó por regresión a partir de los datos obtenidos de porcentajes de inhibición. BHT y té negro se ensayaron también y los resultados obtenidos se utilizaron para propósitos de comparación.

\section{Análisis estadístico}

Todos los datos se obtuvieron por triplicado y los resultados se expresaron como valor medio \pm desviación estándar. El análisis estadístico de los datos se realizó utilizando el software Statgraphic Centurion 15. Las relaciones entre las variables respuesta analizadas se procesaron computando los coeficientes lineales de correlación de Pearson para $p$-valor $<0,05(\mathrm{NC}=95 \%)$. Se obtuvieron ecuaciones que relacionan las variables medidas SGT y con la cantidad de hojas utilizada en la infusión $\left(\mathrm{R}^{2} \geq 95 \%\right)$.

\section{Resultados y Discusión}

\section{Recuperación de SGs en las infusiones}

Los contenidos de SGs de las hojas de las tres variedades de stevia cultivadas en Argentina y estudiadas aquí, se presentan en la Tabla 1. La época de cosecha elegida proporciona los contenidos de SGs más elevados y también la relación $\mathrm{RbA} / \mathrm{Stv}$ más alta para las variedades consideradas $[23,25]$. Cada variedad presenta un perfil de SGs diferente y característico.

Los contenidos de SGs de las infusiones dependiendo de la variedad y de la cantidad de hojas utilizada en la 
infusión se presentan en la Tabla 2. Las variedades de stevia con mayor relación $\mathrm{RbA} / \mathrm{Stv}$ presentan en general reducción de retrogusto persistente en infusiones de stevia $[1,10]$; esto se debe a la reducción del contenido de Stv en las hojas. La relación RbA/Stv en las infusiones fue de 0,6 para SC, de 1,1 para SE y 3,4-3,6 para SK (Tabla 2). Para SK la relación RbA/Stv aumento continuamente con el aumento de la cantidad de hojas utilizada en la infusión $(p$-valor $<0,05)$.

Tabla 1: Contenido de SGs cuantificados en hojas de las variedades SC, SE y SK

\begin{tabular}{|c|c|c|c|c|c|c|}
\hline \multirow{2}{*}{ Variedad } & \multicolumn{4}{|c|}{ SG (\%) ${ }^{a}$} & \multirow{2}{*}{$\begin{array}{l}\text { Contenido } \\
\text { de } \\
\text { humedad } \\
(\%)\end{array}$} & \multirow{2}{*}{$\begin{array}{l}\text { Relación } \\
\text { RbA/Stv }\end{array}$} \\
\hline & Stv & $\mathrm{RbA}$ & $\mathrm{RbC}$ & $\mathrm{SGT}^{\mathrm{b}}$ & & \\
\hline sc & $7,6 \pm 0,3$ & $4,4 \pm 0,1$ & $0,7 \pm 0,1$ & $13,2 \pm 0,2$ & $6,4 \pm 0,2$ & 0,6 \\
\hline SE & $7,4 \pm 0,2$ & $7,8 \pm 0,3$ & $1,1 \pm 0,2$ & $16,1 \pm 0,3$ & $7,6 \pm 0,3$ & 1,1 \\
\hline SK & $3,5 \pm 0,2$ & $11,7 \pm 0,3$ & $1,3 \pm 0,1$ & $16,6 \pm 0,3$ & $6,8 \pm 0,2$ & 3,5 \\
\hline
\end{tabular}

a \%, porcentaje de glicósidos de steviol en las hojas (en base seca); ${ }^{b}$ SGT, suma de los SGs cuantificados

En la Tabla 2 se presenta la recuperación de SGs a partir de las hojas. La recuperación de glicósidos de steviol totales cuantificados (SGT) estuvo entre 13,1-13,3\% en stevia SC, entre $16,8-17,4 \%$ en stevia SE y entre $16,5-16,7 \%$ en stevia SK. Resultados comparables fueron obtenidos anteriormente por otros autores en condiciones similares a la infusión de stevia estudiada aquí $[7,28]$.

Los valores significativamente diferentes en la misma columna (Prueba de ANOVA y prueba de Tukey) presentan diferentes superíndices ( $p$-valor $<0,05) .{ }^{\times}$SGT: suma de los glicósidos de steviol cuantificados. y $\%$ basado en 10 gramos de hojas secas.

\section{Recuperación de sólidos totales}

Los STE comprenden todas las sustancias orgánicas e inorgánicas solubilizadas y en suspensión, recuperadas de las hojas de stevia y presentes en las infusiones [21]. Los contenidos de STE caracterizados en las infusiones realizadas con diferentes pesos de hojas por infusión, se presentan en la Tabla 3. La recuperación de sólidos totales depende de la variedad y del contenido de SGs en las hojas $(p$-valor $<0,05)$. La recuperación de STE resultó mayor a $59 \%$ (en base seca) en las infusiones de stevia de la variedad SK; para SE la recuperación de STE estuvo entre 45,5 y $62,7 \%$ (Tabla 3 ); en el caso de SC la recuperación de sólidos resultó menor (45,5-58,3\%). En general los resultados obtenidos son consistentes con los determinados en estudios anteriores (Tabla 3).

Tabla 2: Concentración de SGs (mg SG por infusión, 200 mL), rendimiento (\% extraído) y recuperación (\% de SGs recuperados a partir de 100 g de hojas secas)

\begin{tabular}{|c|c|c|c|c|c|c|c|c|}
\hline \multirow{2}{*}{$\begin{array}{l}\text { Varie- } \\
\text { dad }\end{array}$} & \multirow{2}{*}{$\begin{array}{l}\text { g por infusión } \\
(200 \mathrm{~mL})\end{array}$} & \multicolumn{4}{|c|}{ Concentración (mg SG/ infusión) } & \multirow{2}{*}{$\begin{array}{c}\text { Rendimiento } \\
\text { (\% SGT) }\end{array}$} & \multirow{2}{*}{$\begin{array}{c}\text { Recuperación } \\
\text { (\% en base } \\
\text { seca) }{ }^{y}\end{array}$} & \multirow{2}{*}{$\begin{array}{l}\text { Relación } \\
\text { RbA/Stv }\end{array}$} \\
\hline & & Stv & RbA & $\mathrm{RbC}$ & SGT ${ }^{x}$ & & & \\
\hline \multirow[t]{6}{*}{ SC } & 0,2 & $14,8 \pm 0,5$ & $8,7 \pm 0,2$ & $1,4 \pm 0,1$ & $24,9 \pm 0,3$ & $99,5 \pm 0,7^{\mathrm{abcd}}$ & $13,3 \pm 0,2^{f}$ & $0,6 \pm 0,0$ \\
\hline & 0,4 & $29,3 \pm 0,8$ & $17,3 \pm 0,6$ & $2,5 \pm 0,1$ & $49,2 \pm 0,1$ & $98,7 \pm 0,2^{\mathrm{bcd}}$ & $13,1 \pm 0,1^{f}$ & $0,6 \pm 0,0$ \\
\hline & 0,8 & $58,0 \pm 1,8$ & $34,6 \pm 1,2$ & $5,8 \pm 0,3$ & $98,4 \pm 0,8$ & $98,7 \pm 0,9^{\mathrm{bcd}}$ & $13,1 \pm 0,1^{f}$ & $0,6 \pm 0,0$ \\
\hline & 1,2 & $89,0 \pm 1,4$ & $52,2 \pm 0,5$ & $8,4 \pm 0,3$ & $149,7 \pm 2,1$ & $100,1 \pm 1,4^{a}$ & $13,3 \pm 0,2^{f}$ & $0,6 \pm 0,0$ \\
\hline & 1,6 & $115,8 \pm 2,4$ & $69,6 \pm 0,9$ & $11,1 \pm 0,1$ & $196,5 \pm 1,7$ & $98,6 \pm 0,8^{\text {cd }}$ & $13,1 \pm 0,2^{f}$ & $0,6 \pm 0,0$ \\
\hline & 2,0 & $148,5 \pm 0,2$ & $87,1 \pm 0,6$ & $14,2 \pm 0,5$ & $249,8 \pm 4,8$ & $99,4 \pm 0,5^{\text {abcd }}$ & $13,3 \pm 0,2^{f}$ & $0,6 \pm 0,0$ \\
\hline \multirow[t]{6}{*}{ SE } & 0,2 & $14,1 \pm 0,2$ & $16,0 \pm 0,1$ & $1,9 \pm 0,0$ & $32,0 \pm 0,2$ & $100,0 \pm 0,3^{a}$ & $17,4 \pm 0,2^{\mathrm{a}}$ & $1,1 \pm 0,0$ \\
\hline & 0,4 & $28,3 \pm 0,2$ & $29,6 \pm 1,3$ & $3,9 \pm 0,1$ & $61,7 \pm 1,5$ & $98,6 \pm 1,3^{\text {cd }}$ & $16,8 \pm 0,2^{\text {cd }}$ & $1,0 \pm 0,0$ \\
\hline & 0,8 & $56,3 \pm 0,3$ & $60,9 \pm 1,4$ & $7,6 \pm 0,2$ & $124,7 \pm 1,7$ & $99,8 \pm 0,3^{\mathrm{abc}}$ & $16,9 \pm 0,2^{b c}$ & $1,1 \pm 0,0$ \\
\hline & 1,2 & $85,1 \pm 2,0$ & $90,5 \pm 1,1$ & $11,5 \pm 0,5$ & $187,1 \pm 2,6$ & $100,0 \pm 0,1^{\mathrm{a}}$ & $17,0 \pm 0,2^{\mathrm{bc}}$ & $1,1 \pm 0,0$ \\
\hline & 1,6 & $114,8 \pm 1,1$ & $121,6 \pm 1,2$ & $16,1 \pm 0,4$ & $252,33 \pm 0,5$ & $100,0 \pm 0,2^{\mathrm{a}}$ & $17,0 \pm 0,2^{\mathrm{ab}}$ & $1,0 \pm 0,0$ \\
\hline & 2,0 & $141,6 \pm 1,1$ & $149,9 \pm 0,9$ & $19,6 \pm 0,7$ & $310,0 \pm 1,1$ & $99,9 \pm 0,2^{\mathrm{ab}}$ & $16,9 \pm 0,2^{b c}$ & $1,1 \pm 0,0$ \\
\hline \multirow[t]{6}{*}{ SK } & 0,2 & $6,4 \pm 0,1$ & $22,4 \pm 0,4$ & $2,4 \pm 0,2$ & $31,1 \pm 0,5$ & $99,5 \pm 0,9$ abcd & $16,7 \pm 0,2^{\text {cd }}$ & $3,5 \pm 0,1$ \\
\hline & 0,4 & $12,8 \pm 0,3$ & $43,5 \pm 1,1$ & $4,7 \pm 0,3$ & $61,2 \pm 1,2$ & $98,5 \pm 1,4^{d}$ & $16,0 \pm 0,2^{\mathrm{e}}$ & $3,4 \pm 0,1$ \\
\hline & 0,8 & $24,8 \pm 0,8$ & $89,8 \pm 2,0$ & $9,5 \pm 0,4$ & $124,2 \pm 1,9$ & $99,4 \pm 1,0^{\text {abcd }}$ & $16,7 \pm 0,2^{\text {cd }}$ & $3,6 \pm 0,1$ \\
\hline & 1,2 & $37,5 \pm 0,4$ & $134,9 \pm 2,1$ & $14,0 \pm 0,2$ & $186,4 \pm 1,9$ & $99,7 \pm 0,4^{\mathrm{abcd}}$ & $16,7 \pm 0,2^{c d}$ & $3,6 \pm 0,1$ \\
\hline & 1,6 & $49,6 \pm 1,6$ & $180,4 \pm 0,1$ & $19,3 \pm 0,6$ & $249,4 \pm 0,8$ & $100,0 \pm 0,3^{a}$ & $16,7 \pm 0,2^{\text {cd }}$ & $3,6 \pm 0,1$ \\
\hline & 2,0 & $62,4 \pm 1,4$ & $222,1 \pm 0,9$ & $24,0 \pm 0,2$ & $309,7 \pm 1,7$ & $99,3 \pm 0,6^{\mathrm{abcd}}$ & $16,7 \pm 0,2^{\mathrm{e}}$ & $3,6 \pm 0,1$ \\
\hline $\begin{array}{l}\text { Valores de } \\
\text { referencia }[7,28]\end{array}$ & 5,0 & & & & & $90,0-99,3$ & $7,2-15,5$ & $0,4-4,2$ \\
\hline
\end{tabular}


Tabla 3: Concentración (mg STE/ infusión) y recuperación (\% en base seca) en infusiones de stevia dependiendo de la variedad

\begin{tabular}{|c|c|c|c|}
\hline Variedad & $\begin{array}{l}\text { g de hoja por } \\
\text { infusión }\end{array}$ & $\begin{array}{l}\text { Concentración } \\
\text { (mg/ infusión) }\end{array}$ & $\begin{array}{l}\text { Recuperación (\% } \\
\text { en base seca) }\end{array}$ \\
\hline \multirow[t]{6}{*}{ SC } & 0,2 & $85,3 \pm 17,0$ & $45,5 \pm 9,1^{c}$ \\
\hline & 0,4 & $218,7 \pm 13,3$ & $58,3 \pm 3,5^{\mathrm{ab}}$ \\
\hline & 0,8 & $426,0 \pm 26,2$ & $56,8 \pm 3,5^{\mathrm{ab}}$ \\
\hline & 1,2 & $648,7 \pm 68,2$ & $57,7 \pm 6,1^{\mathrm{ab}}$ \\
\hline & 1,6 & $832,7 \pm 39,0$ & $55,5 \pm 2,6^{\mathrm{ab}}$ \\
\hline & 2,0 & $1027,3 \pm 75,6$ & $54,8 \pm 4,0^{\mathrm{ab}}$ \\
\hline \multirow[t]{6}{*}{ SE } & 0,2 & $84,7 \pm 19,4$ & $45,2 \pm 10,4^{c}$ \\
\hline & 0,4 & $200,8 \pm 26,8$ & $53,5 \pm 7,1 b^{c}$ \\
\hline & 0,8 & $462,0 \pm 51,9$ & $61,6 \pm 6,9 \mathrm{ab}$ \\
\hline & 1,2 & $705,4 \pm 74,0$ & $62,7 \pm 6,6^{\mathrm{ab}}$ \\
\hline & 1,6 & $910,0 \pm 42,0$ & $60,7 \pm 2,8^{\mathrm{ab}}$ \\
\hline & 2,0 & $1114,0 \pm 48,5$ & $59,4 \pm 2,6^{\text {ab }}$ \\
\hline \multirow[t]{8}{*}{ SK } & 0,2 & $112,7 \pm 4,2$ & $60,2 \pm 2,2^{b}$ \\
\hline & 0,4 & $222,9 \pm 27,6$ & $59,6 \pm 7,4^{\mathrm{ab}}$ \\
\hline & 0,8 & $473,3 \pm 32,5$ & $63,3 \pm 4,3^{\text {a }}$ \\
\hline & 1,2 & $703,3 \pm 42,4$ & $62,7 \pm 3,8^{b}$ \\
\hline & 1,6 & $928,7 \pm 70,2$ & $62,1 \pm 4,7^{\mathrm{ab}}$ \\
\hline & 2,0 & $1100,0 \pm 76,0$ & $58,8 \pm 4,1^{1 \mathrm{ab}}$ \\
\hline & $\begin{array}{l}\text { Té negro ( } 2 \text { g } \\
\text { por infusión) }\end{array}$ & $852,1 \pm 4,3$ & $44,9 \pm 1,2$ \\
\hline & $\begin{array}{l}\text { Valores de } \\
\text { referencia }{ }^{x}\end{array}$ & $1084-1236$ & $27,6-61,9$ \\
\hline \multicolumn{4}{|c|}{$\begin{array}{l}\text { Los valores significativamente diferentes en la misma columna (Prueba de ANOVA } \\
\text { y prueba de Tukey) presentan diferentes superíndices }(p-v a l o r<0,05) .{ }^{\times} \text {sustancias } \\
\text { solubles en agua y carbohidratos calculados por diferencia }[13-14,16,18,21,25]\end{array}$} \\
\hline
\end{tabular}

La biosíntesis de fitoquímicos en stevia depende de diversos factores de pre-cosecha incluyendo variedad, condiciones climáticas y prácticas culturales [21, 23, 36]. Las condiciones de cosecha elegidas proporcionan elevados contenidos de SGs y también recuperaciones elevadas de sólidos totales. Por otro lado, la recuperación de sólidos aumenta en el mismo orden que el contenido de SGs en cada variedad, a saber $\mathrm{SC}<\mathrm{SE}<\mathrm{SK}$.

\section{Recuperación de compuestos fenólicos y de taninos}

En estudios anteriores se determinó que el agua es el solvente más adecuado para recuperar compuestos fenólicos y SGs desde hojas de stevia [25]. Los contenidos de FT de las infusiones se presentan en la Tabla 4. Los resultados obtenidos en este estudio son concordantes con los determinados en otros cultivares de stevia y están en el mismo orden en comparación con té negro (Tabla 4). Además, infusiones de SK proporcionan valores mayores de FT seguidos por SE y SC ( $p$-valor $<0,05)$.

Los contenidos de taninos totales determinados en las infusiones (TT), también se presentan en la Tabla 4; como puede verse, en comparación con té negro los contenidos de taninos son significativamente menores. Diferente a los compuestos fenólicos totales, en los taninos se observa el siguiente orden: $\mathrm{SE}<\mathrm{SK}<\mathrm{SC}$.

Como ocurre con los contenidos de SGs, los contenidos de FT son afectados por diversos factores que incluyen momento de cosecha, condiciones agro-ecológicas y climáticas, condiciones de cultivo, variedad, tratamientos pos-cosecha y finalmente el método y solvente extracción $[21,23,31,36]$. En relación a la variedad, infusiones de stevia con mayor contenido total de SGs y con mayor relación $\mathrm{RbA} / \mathrm{Stv}$ proporcionaron mayores contenidos de compuestos fenólicos totales. Sin embargo un comportamiento diferente se observó en los contenidos de taninos; en este caso infusiones de SE con una relación $\mathrm{RbA} / \mathrm{Stv}$ de 1,1 proporcionan contenidos taninos totales mayores (Tabla 4).

Tabla 4: Compuestos fenólicos y taninos, concentración (mg AGE por infusión, 200 mL) y recuperación (\% AGE recuperados a partir de $100 \mathrm{~g}$ de hojas secas)

\begin{tabular}{|c|c|c|c|c|c|}
\hline \multirow[t]{2}{*}{ Variedad } & \multirow{2}{*}{$\begin{array}{l}\text { g de hoja } \\
\text { por infusión }\end{array}$} & \multicolumn{2}{|c|}{$\begin{array}{l}\text { Concentración (mg } \\
\text { AGE/ infusión) }\end{array}$} & \multicolumn{2}{|c|}{$\begin{array}{l}\text { Recuperación (\% AGE } \\
\text { en base seca) }\end{array}$} \\
\hline & & FT & TT & FT & TT \\
\hline \multirow[t]{6}{*}{ SC } & 0,2 & $4,1 \pm 0,9$ & $0,2 \pm 0,1$ & $2,2 \pm 0,5^{j}$ & $0,1 \pm 0,1^{\mathrm{h}}$ \\
\hline & 0,4 & $13,8 \pm 0,9$ & $1,7 \pm 0,2$ & $3,7 \pm 0,3^{i}$ & $0,5 \pm 0,1^{\text {gh }}$ \\
\hline & 0,8 & $34,6 \pm 4,3$ & $6,3 \pm 0,8$ & $4,6 \pm 0,6^{f g}$ & $0,8 \pm 0,1^{\text {ef }}$ \\
\hline & 1,2 & $54,0 \pm 3,0$ & $16,3 \pm 1,5$ & $4,8 \pm 0,3^{\mathrm{fg}}$ & $0,8 \pm 0,2^{\text {efg }}$ \\
\hline & 1,6 & $67,0 \pm 2,5$ & $21,2 \pm 1,2$ & $4,5 \pm 0,2^{g h}$ & $0,9 \pm 0,1^{\text {def }}$ \\
\hline & 2,0 & $86,9 \pm 3,8$ & $25,2 \pm 4,4$ & $4,6 \pm 0,2^{\text {fgh }}$ & $0,8 \pm 0,1^{\text {efg }}$ \\
\hline \multirow[t]{6}{*}{ SE } & 0,2 & $7,9 \pm 0,5$ & $1,1 \pm 0,9$ & $4,3 \pm 0,3^{h}$ & $0,6 \pm 0,5^{f g}$ \\
\hline & 0,4 & $20,1 \pm 0,9$ & $3,6 \pm 1,3$ & $5,5 \pm 0,2^{\text {cde }}$ & $1,0 \pm 0,4^{\text {de }}$ \\
\hline & 0,8 & $41,0 \pm 2,6$ & $9,3 \pm 2,1$ & $5,6 \pm 0,4^{\mathrm{cd}}$ & $1,2 \pm 0,3^{\mathrm{abcc}}$ \\
\hline & 1,2 & $63,8 \pm 2,2$ & $16,4 \pm 1,9$ & $5,8 \pm 0,2^{c}$ & $1,4 \pm 0,2^{\mathrm{a}}$ \\
\hline & 1,6 & $82,3 \pm 3,3$ & $19,7 \pm 2,2$ & $5,6 \pm 0,2^{c d}$ & $1,3 \pm 0,1^{1 \mathrm{abc}}$ \\
\hline & 2,0 & $97,3 \pm 4,1$ & $26,4 \pm 2,0$ & $5,3 \pm 0,2^{\text {de }}$ & $1,4 \pm 0,1^{\mathrm{ab}}$ \\
\hline \multirow[t]{6}{*}{ SK } & 0,2 & $9,3 \pm 0,1$ & $0,4 \pm 0,1$ & $5,0 \pm 0,1^{\text {ef }}$ & $0,2 \pm 0,1^{\mathrm{h}}$ \\
\hline & 0,4 & $24,1 \pm 1,4$ & $3,2 \pm 1,3$ & $6,5 \pm 0,4^{b}$ & $0,9 \pm 0,3^{\text {ef }}$ \\
\hline & 0,8 & $56,1 \pm 2,5$ & $7,5 \pm 1,6$ & $7,5 \pm 0,3^{\mathrm{a}}$ & $1,0 \pm 0,2^{\text {cde }}$ \\
\hline & 1,2 & $87,9 \pm 2,4$ & $12,0 \pm 2,5$ & $7,9 \pm 0,2^{\mathrm{a}}$ & $1,1 \pm 0,2^{\text {bcde }}$ \\
\hline & 1,6 & $113,2 \pm 2,7$ & $14,5 \pm 3,4$ & $7,6 \pm 0,2^{\mathrm{a}}$ & $1,0 \pm 0,2^{\text {cde }}$ \\
\hline & 2,0 & $138,9 \pm 3,1$ & $17,8 \pm 3,5$ & $7,6 \pm 0,2^{\mathrm{a}}$ & $1,0 \pm 0,2^{\text {cde }}$ \\
\hline \multicolumn{2}{|c|}{ Té negro } & $109,2 \pm 1,4$ & $37,9 \pm 3,6$ & $5,1 \pm 0,1$ & $1,8 \pm 0,2$ \\
\hline \multicolumn{2}{|c|}{$\begin{array}{c}\text { FT determinados } \\
\text { previamente en extractos } \\
\text { acuosos }[26,28,37]\end{array}$} & & & $2,7-9,3$ & \\
\hline
\end{tabular}

os valores significativamente diferentes en la misma columna (Prueba de ANOVA y prueba de Tukey) presentan diferentes superíndices $(p$-valor $<0,05)$

\section{Actividad antiradicalaria de las infusiones}

Se investigó la actividad antiradicalaria en las infusiones de stevia frente a $\mathrm{DPPH} \bullet$. Los resultados obtenidos incluyendo los controles BHT y té negro se presentan en la Figura 1. Las infusiones de las tres variedades exhibieron actividad antioxidante elevada con valores de $\mathrm{IC}_{50}=$ $18,6-27,7 \mu \mathrm{g} / \mathrm{mL}$. El orden de actividad observada (SK $>\mathrm{SC}>\mathrm{SE}$ ) es consistente con los contenidos de FT de las infusiones de SK, sin embargo la actividad antiradicalaria fue mayor en SC (con FT menor) comparando con SE.

Diversos tipos de compuestos presentes en las infusiones pueden contribuir a la actividad antioxidante observada $[25,32,38]$. Sumado a la composición de las hojas, diversos factores incluyendo variedad, labores culturales, 
tratamientos pos-cosecha y método de extracción pueden influir los resultados de esa actividad antioxidante.

Los resultados obtenidos para infusiones de stevia estuvieron en el mismo orden del té negro y BHT (Figura 1), y son consistentes con resultados determinados previamente en extractos de stevia cosechada en verano [37].

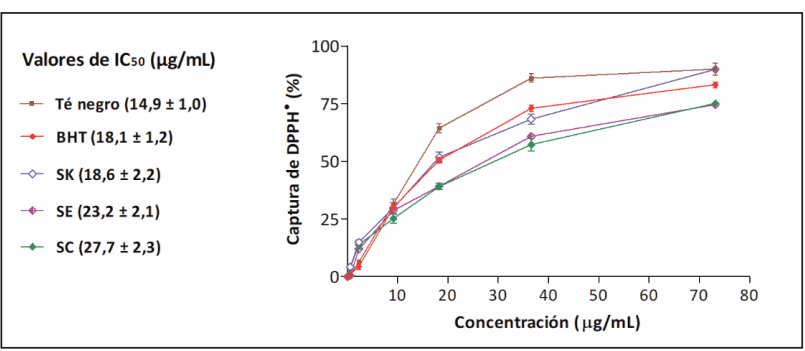

Figura 1: Actividad antiradicalaria de infusiones de stevia de las variedades SK, SE, SC frente al radical DPPH•

\section{Correlaciones entre SGT, FT, TT y STE}

De manera general el aumento del contenido de SGs y de FT se ve reflejado en un aumento proporcional del contenido de sólidos totales en las infusiones. Los resultados de concentraciones obtenidas se utilizaron para investigar las correlaciones existentes entre los grupos fitoquímicos investigados en las infusiones de stevia. Se listan en la Tabla 5 los coeficientes de correlación de Pearson determinados al investigar correlaciones entre fitoquímicos de las infusiones.

La presencia de correlación se reportó previamente entre Stv y carbohidratos solubles totales en extractos acuosos de stevia, también entre SGT y STE [13, 22]. En otro estudio Tavarini y colaboradores [32], establecieron que existe correlación entre la actividad antioxidante y los compuestos fenólicos y flavonoides en extractos de stevia; en este mismo estudio se encontró baja correlación entre la actividad antioxidante y los contenidos de Stv y RbA en los mismos extractos.

En el presente estudio, se determinó una elevada correlación entre RbA y STE, entre RbC y STE (Tabla 5, Figura 2). También se estableció correlación elevada entre RbA y FT, entre RbC y FT. Por su parte Stv se correlacionó mejor con TT.

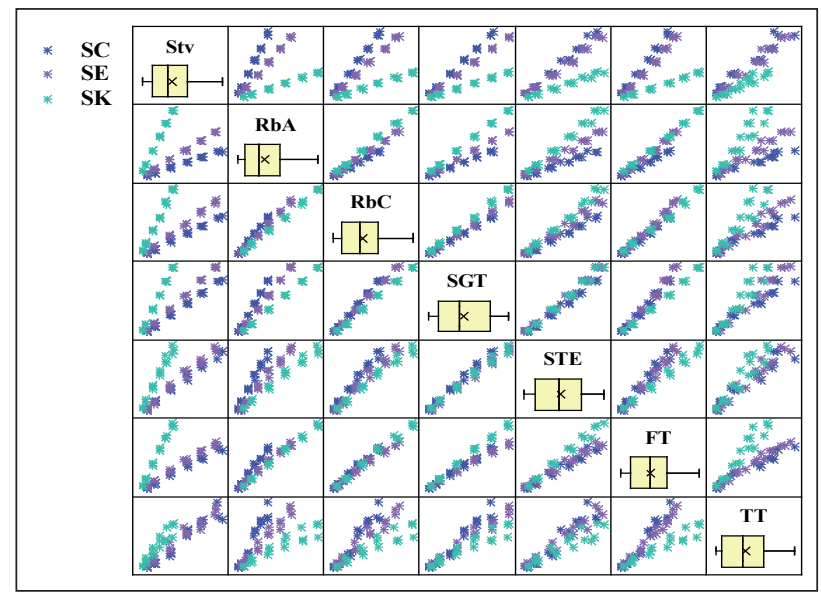

Figura 2: Correlaciones entre las variables determinadas en infusiones de stevia dependiendo de la variedad

\section{Contenido de SGs edulcorantes y FT antioxidantes en infusiones de stevia}

La dependencia entre los contenidos de SGT y las cantidades de hojas utilizadas para endulzar una infusión para las variedades de stevia estudiadas aquí se da por:

$$
\begin{array}{ll}
S G T_{S C}=105,2 . \text { st } & R^{2}=98,89 \% \\
S G T_{S E}=122,8 . \text { st } & R^{2}=98,93 \% \\
S G T_{S K}=156,6 . \text { st } & R^{2}=99,62 \%
\end{array}
$$

\begin{tabular}{|c|c|c|c|c|c|c|c|}
\hline Variable & Stv & RbA & $\mathrm{RbC}$ & SGT & STE & FT & \\
\hline Stv & 1,0000 & & & & & & \\
\hline $\mathrm{RbA}$ & $\begin{array}{c}0,4192 \\
(0,0016) a\end{array}$ & 1,0000 & & & & & \\
\hline $\mathrm{RbC}$ & $\begin{array}{c}0,6170 \\
(0,0000)\end{array}$ & $\begin{array}{c}0,9714 \\
(0,0000)\end{array}$ & 1,0000 & & & & \\
\hline SGT & $\begin{array}{c}0,7774 \\
(0,0000)\end{array}$ & $\begin{array}{c}0,8969 \\
(0,0000)\end{array}$ & $\begin{array}{c}0,9736 \\
(0,0000)\end{array}$ & 1,0000 & & & \\
\hline STE & $\begin{array}{c}0,8141 \\
(0,0000)\end{array}$ & $\begin{array}{c}0,8501 \\
(0,0000)\end{array}$ & $\begin{array}{c}0,9429 \\
(0,0000)\end{array}$ & $\begin{array}{c}0,9854 \\
(0,0000)\end{array}$ & 1,0000 & & \\
\hline FT & $\begin{array}{c}0,5957 \\
(0,0000)\end{array}$ & $\begin{array}{c}0,9649 \\
(0,0000)\end{array}$ & $\begin{array}{c}0,9878 \\
(0,0000)\end{array}$ & $\begin{array}{c}0,9587 \\
(0,0000)\end{array}$ & $\begin{array}{c}0,9381 \\
(0,0000)\end{array}$ & 1,0000 & \\
\hline$T T$ & $\begin{array}{c}0,9197 \\
(0,0000)\end{array}$ & $\begin{array}{c}0,6668 \\
(0,0000)\end{array}$ & $\begin{array}{c}0,8070 \\
(0,0000)\end{array}$ & $\begin{array}{c}0,9096 \\
(0,0000)\end{array}$ & $\begin{array}{c}0,9214 \\
(0,0000)\end{array}$ & $\begin{array}{c}0,8076 \\
(0,0000)\end{array}$ & 1,0000 \\
\hline
\end{tabular}

Tabla 5: Coeficientes de correlación de Pearson entre STE (mg/ infusión), SGs (mg/ infusión), FT (mg/ infusión) y TT (mg/ infusión), dependiendo de la variedad

a $p$-valor entre paréntesis $(\mathrm{NC}=95 \%, \mathrm{n}=54)$ 
Donde SGT es la concentración de glicódidos de steviol en una tasa de infusión (mg/infusión) y st es la cantidad de hojas utilizada para ese fin (gramos). Las Ec. (1), (2) y (3) proporcionan una predicción teórica de los contenidos de SGs edulcorantes en infusiones dependiendo de la variedad (despreciando el efecto de la humedad en el cálculo).

Del mismo modo los compuestos fenólicos totales pueden obtenerse a partir de los g de hojas que se utilicen para endulzar la infusión (st) a partir de:

$$
\begin{array}{ll}
F T_{S C}=43,9 . \mathrm{st} & R^{2}=97,57 \% \\
F T_{S E}=51,2 . \mathrm{st} & R^{2}=98,59 \% \\
F T_{S K}=73,5 . \mathrm{st} & R^{2}=99,30 \%
\end{array}
$$

Donde FT es la concentración de compuestos fenólicos totales aportada por stevia en una tasa de infusión (mg AGE/infusión). Las Ec. (1) a (6) proporcionan una predicción teórica de los contenidos de SGs edulcorantes y de compuestos fenólicos antioxidantes en infusiones de stevia dependiendo de la variedad.

Las ecuaciones establecidas son válidas para las variedades de stevia estudiadas aquí y cuando la cosecha se realizó en verano coincidente con los máximos en los contenidos de SGs edulcorantes y con los niveles más altos de RbA en las hojas de la planta. Con respecto a los contenidos de taninos, son necesarios mayores estudios a fin de poder establecer la naturaleza de la variabilidad determinada en este estudio.

Finalmente, con respecto al poder edulcorante relativo, tomando para los SGs de stevia un poder edulcorante de 200 con respecto a sacarosa $[4,13,18]$ y utilizando estimaciones basadas en las Ec. (1) a (3), tendremos que 0,2 - 0,4 $\mathrm{g}$ de hojas molidas de SK resultarán adecuados para endulzar una infusión de $200 \mathrm{~mL}\left(\mathrm{o} \mathrm{cm}^{3}\right) ; 0,3$ - 0,5 g de hojas de SE y 0,4 - 0,6 g de SC para el mismo propósito. Esta estimación del poder edulcorante de las hojas de stevia resulta satisfactoria si se utilizan bajos contenidos de hojas de stevia en cada infusión [39, 40], como los empleados en este estudio. En cuanto a los contenidos de FT en la infusión dependiendo de la variedad, también estos pueden expresarse en base a la cantidad de hojas utilizada en la infusión (Ec. 4 a 6).

Además del perfil característico de SGs de cada variedad de stevia, otros fitoquímicos presentes en las hojas pueden influenciar la percepción sensorial de los preparados de stevia, incluyendo los compuestos fenólicos y en especial los taninos [37, 40]. Tal efecto será estudiado en investigaciones futuras.

\section{Conclusiones}

En este estudio se determinó la recuperación de sólidos totales, de glicósidos de steviol edulcorantes, de compuestos fenólicos antioxidantes y de taninos totales, en infusiones de $S$. rebaudiana de tres variedades cultivadas en Argentina. Se encontró que infusiones preparadas con variedades con mayor contenido de glicósidos de steviol endulzantes proporcionan también contenidos de compuestos fenólicos mayores. Se determinó que Rebaudiósido A y C correlacionan con los compuestos fenólicos y que Esteviósido correlaciona con los taninos en las infusiones. Se establecieron ecuaciones para estimar los contenidos de glicósidos de steviol edulcorantes y compuestos fenólicos en las infusiones de stevia de las tres variedades investigadas.

Las infusiones de stevia de las variedades cultivadas en Argentina son una rica fuente de compuestos fenólicos antioxidantes.

\section{Agradecimientos}

Liliana S. Celaya agradece al Consejo Nacional de Investigaciones Científicas y Técnicas por la Beca PosDoctoral otorgada, CONICET Res. DN594/17 (Projecto UNaM 16Q624, N. Kolb). Los autores agradecen a Cristina E. Mayol por su apoyo y colaboración.

\section{Referencias}

1. Celaya, L.S.; Kolb, E.; Kolb, N. Solubility of Stevioside and Rebaudioside A in water, ethanol and their binary mixtures. International Journal of Food Studies 5:158-166. 2016.

2. Joint FAO/WHO Expert Committee on Food Additives 2008. Steviol Glycosides. Chemical and Technical Assessment (CTA, 2007) for the 68th JECFA [en línea] Disponible en: www.fao.org/fileadmin/templates/agns/pdf/jecfa/ cta. Acceso 16 Junio 2010.

3. Código Alimentario Argentino, Cap. XVIII, Aditivos Alimentarios. Artículo 1192 tris [Actualización 01/2014]. [en línea] Disponible www.anmat.gov Acceso 9 Mayo 2016.

4. Prakash, I.; Chaturvedula, v.S.P. Steviol Glycosides: Natural Non-Caloric Sweeteners, en: Mérillon, J.-M., Ramawat, K.G. (Eds.), Sweeteners: Pharmacology, Biotechnology, and Applications. Springer International Publishing AG, 101-128. 2016.

5. Wölwer-Rieck, U. The leaves of Stevia rebaudiana (Bertoni), their constituents and the analyses thereof: $A$ review. Journal of Agricultural and Food Chemistry 60:886-895. 2012.

6. Bender, C. Stevia rebaudiana's Antioxidant Properties, en: Mérillon, J.-M., Ramawat, K.G. (Eds.), Sweeteners: Pharmacology, Biotechnology, and Applications. Springer International Publishing AG, 349-375. 2016.

7. Jentzer, J., Alignan, M., Vaca-garcia, C., Rigal, L., Vilarem, G. Response surface methodology to optimise Accelerated Solvent Extraction of steviol glycosides from Stevia rebaudiana Bertoni leaves. Food Chemistry 166:561567. 2015. 
8. Kolb, N.; Herrera, J.L.; Ferreyra, D.J.; Uliana, R.F. Analysis of sweet diterpene glycosides from Stevia rebaudiana: Improved HPLC method. Journal of Agricultural and Food Chemistry 49:4538-4541. 2001.

9. Yadav, Singh, S.; Dhyani, D., Ahuja, P.S. A review on the improvement of stevia [Stevia rebaudiana (Bertoni)]. Canadian Journal of Plant Science 91:1-27. 2011.

10. Dacome, A.S., Da Silva, C.C., Da Costa, C.E.M., Fontana, J.D., Adelmann, J., Da Costa, S.c. Sweet diterpenic glycosides balance of a new cultivar of Stevia rebaudiana (Bert.) Bertoni: Isolation and quantitative distribution by chromatographic, spectroscopic, and electrophoretic methods. Process Biochemistry 40:3587-3594. 2005.

11. Dewis, M.L.; Kraut, K.J.; Merritt, D.; Salemme, R.F. Methods for using rebaudioside C as a flavor enhancer. United States Patent Application US20138524785. 2013.

12. Brower, R.J.; Carlson, T.L.; Dang, B.; Gonzalez, M.; Kennedy, M.M.; Knutson, N.E. Stevia plants with an increased Rebaudioside D content. United States Patent Application US20160021918A1. 2016.

13. Abou-Arab, E.A.; Abou-Arab, A.A.; Abu-Salem, M.F. Physico-chemical assessment of natural sweeteners steviosides produced from Stevia rebaudiana Bertoni plant. African Journal of Food Science 4:269-281. 2010.

14. Chabot, S.; Beaulieu, M. Extraction method for providing an organic certifiable Stevia rebaudiana extract. United States Patent Application, US20130108718. 2013.

15. Fuh w-S y Chiang B-H. Purification of Steviosides by Membrane and Ion Exchange Processes. Journal of Food Science 55:1454-1457. 1990.

16. Liu J.; Li J.; Tang J. Ultrasonically assisted extraction of total carbohydrates from Stevia rebaudiana Bertoni and identification of extracts. Food and Bioproducts Processing 88:215-221. 2010.

17. Martins P.M.; Lanchote A.D.; Thorat B.N.; Freitas L.A.P. Turboextraction of glycosides from Stevia rebaudiana using a fractional factorial design. Revista Brasileira de Farmacognosia 27:510-518. 2017.

18. Celaya, L.; Cabana, R.; Viturro, C.; Molina, A.; Kolb, N., 2012. Infusión de Stevia rebaudiana Bertoni: sólidos totales, esteviol glicósidos endulzantes y actividad antioxidante. IV Congreso Internacional de Ciencia y Tecnología de los Alimentos CICyTAC 2012; Noviembre de 2012, Córdoba, Argentina. Análisis físicos, químicos y sensoriales, 92-97. 2012.

19. Ramesh, K., Nima, v.S., Megeji W. Cultivation of Stevia [Stevia rebaudiana (Bert.) Bertoni]: A Comprehensive Review. Advances in Agronomy 89:137-177. 2006.

20. Yoda S.K.; Marques M.O.M.; Petenate A.J.; Meireles M.A.A. Supercritical fluid extraction from Stevia rebaudiana Bertoni using $\mathrm{CO} 2$ and $\mathrm{CO} 2+$ water : extraction kinetics and identification of extracted components. Journal of Food Engineering 57:125-134. 2003.

21. Celaya, L. Optimización de la extracción de esteviolglicósidos de Stevia rebaudiana Bertoni. Tesis de Maes- tría, Universidad Nacional de Misiones, Posadas, Argentina. 2013.

22. Nishiyama, P.; Kusumoto, I.T.; Costa, S.C.; Alvarez, M.; Vieira, L.G.E. Correlation between Total Carbohydrate Content and Stevioside Content in Stevia Rebaudiana leaves. Arq Biol Technol 34:3-4. 1991.

23. Taiariol D., Molas M., Lezcano J., Leiva N. Variación estacional de grados Brix en cuatro variedades de Stevia rebaudiana, en EEA INTA Bella Vista, Corrientes. Ciencia y Tecnología de los Cultivos Industriales 6:70-73. 2014.

24. Periche, A.; Koutsidis, G.; Escriche, I. Composition of Antioxidants and Amino Acids in Stevia Leaf Infusions. Plant Foods for Human Nutrition 69:1-7. 2014.

25. Zaidan U.H.; Zen N.I.M.; Amran N.A.; Shamsi S.; Gani S.S.A. Biochemical evaluation of phenolic compounds and steviol glycoside from Stevia rebaudiana extracts associated with in vitro antidiabetic potential. Biocatalysis and Agricultural Biotechnology 18:101049. 2019.

26. Ciulu, M.; Quirantes-Piné, R.; Spano, N.; Sanna, G.; Borrás-Linares, I.; Segura-Carretero, A. Evaluation of new extraction approaches to obtain phenolic compound-rich extracts from Stevia rebaudiana Bertoni leaves. Industrial Crops and Products 108:106-112. 2017.

27. Karaköse, H.; Müller A.; Kuhnert N. Profiling and Quantification of Phenolics in Stevia rebaudiana Leaves. Journal of Agricultural and Food Chemistry 63:9188-9198. 2015.

28. Periche, A.; Castelló, M.L.; Heredia, A.; Escriche, I. Influence of Extraction Methods on the Yield of Steviol Glycosides and Antioxidants in Stevia rebaudiana Extracts. Plant Foods for Human Nutrition 70:119-127. 2015.

29. Tavarini, S.; Sgherri, C.; Ranieri, A.M.; Angelini L.G. Effect of nitrogen fertilization and harvest time on steviol glycosides, flavonoid composition and antioxidant properties in Stevia rebaudiana Bertoni. Journal of Agricultural and Food Chemistry 63:7041-7050. 2015.

30. Verma, A.; Singh, A. Optimization and Quality Assessment of Low-Calorie Herbal Health Tea Sweetened with Stevia (Stevia rebaudiana). Journal of Medical Research and Development 3:134-137. 2014.

31. Rao, A.B., Prasad, E., Roopa, G., Sridhar, S., Ravikumar, Y.V.L. Simple extraction and membrane purification process in isolation of steviosides with improved organoleptic activity. Advances in Bioscience and Biotechnology 3:327-335. 2012.

32. Tavarini S.; Angelini L.G. Stevia rebaudiana Bertoni as a source of bioactive compounds: the effect of harvest time, experimental site and crop age on steviol glycoside content and antioxidant properties. Journal of the Science of Food and Agriculture 93:2121-2129. 2013.

33. Celaya L.S.; Viturro C.I.; Silva L.R.; Moreno S. Natural antioxidants isolated from Schinus areira leaves by ultrasound-assisted extraction. International Journal of Food Studies 5:1-9. 2016. 
34. Azrul, L.M.; Nurulaini, R.; Adzemim, M.A.; Marina, H.; Effendy, A.W.M. Tannins Quantification in Terminalia catappa Leaves Extract and Antihelmenthic Potential Evaluation. Journal of Natural Products 7:98-103. 2014.

35. Celaya, L.; Viturro, C.; Silva, L.R. Chemical Composition and Biological Prospects of Essential Oils and Extracts of Aphyllocladus spartioides Growing in Northwest Argentina. Chemistry and Biodiversity 14:e1600227. 2017.

36. Angelini, L.G.; Martini, A.; Passera, B.; Tavarini S. Cultivation of Stevia rebaudiana Bertoni and Associated Challenges. Mérillon, J.-M. \& Ramawat, K.G. Eds., Sweeteners: Pharmacology, Biotechnology, and Applications. Springer International Publishing AG, 35-85. 2016.

37. Kaushik, R.; Pradeep, N.; Vamshi, v.; Geetha, M.; Usha A. Nutrient composition of cultivated stevia leaves and the influence of polyphenols and plant pigments on sensory and antioxidant properties of leaf extracts. Journal of Food Science and Technology 47:27-33. 2008.

38. Oliveira, A.P.; Pereira, J.A.; Andrade, P.B.; Valentão, P.; Seabra, R.M.; Silva, B.M. Organic acids composition of Cydonia oblonga Miller leaf. Food Chemistry 111:393-399. 2008.

39. Cardello, H.M.A.B.; Da Silva, M.A.P.A.; Damasio, M.H. Measurement of the relative sweetness of stevia extract, aspartame and cyclamate/saccharin blend as compared to sucrose at different concentrations. Plant Foods for Human Nutrition 54:119-130. 1999.

40. Formigoni, M.; Milani, P.G.; Da-Silva Avíncola, A.; Dos-Santos, V.J.; Benossi, L.; Dacome, A.S.; Pilau, E.J.; Da-Costa, S.C. Pretreatment with ethanol as an alternative to improve steviol glycosides extraction and purification from a new variety of stevia. Food Chemistry 241:452-459. 2018. 